\title{
The distribution pattern of proliferating cell nuclear antigen in the nuclei of Leishmania donovani
}

\author{
Correspondence \\ Swati Saha \\ ss5gp@yahoo.co.in
}

Received 31 July 2009

Accepted 27 August 2009

\author{
Devanand Kumar, ${ }^{1}$ Neha Minocha, ${ }^{1}$ Kalpana Rajanala ${ }^{2}$ and Swati Saha ${ }^{1}$ \\ ${ }^{1}$ Department of Microbiology, University of Delhi South Campus, Benito Juarez Road, New Delhi \\ 110021, India \\ ${ }^{2}$ National Institute of Immunology, Aruna Asaf Ali Marg, New Delhi 110067, India
}

DNA replication in eukaryotes is a highly conserved process marked by the licensing of multiple origins, with pre-replication complex assembly in G1 phase, followed by the onset of replication at these origins in $\mathrm{S}$ phase. The two strands replicate by different mechanisms, and DNA synthesis is brought about by the activity of the replicative DNA polymerases Pol $\delta$ and Pol $\varepsilon$. Proliferating cell nuclear antigen (PCNA) augments the processivity of these polymerases by serving as a DNA sliding clamp protein. This study reports the cloning of PCNA from the protozoan Leishmania donovani, which is the causative agent of the systemic disease visceral leishmaniasis. PCNA was demonstrated to be robustly expressed in actively proliferating $L$. donovani promastigotes. We found that the protein was present primarily in the nucleus throughout the cell cycle, and it was found in both proliferating procyclic and metacyclic promastigotes. However, levels of expression of PCNA varied through cell cycle progression, with maximum expression evident in $\mathrm{G} 1$ and $\mathrm{S}$ phases. The subnuclear pattern of expression of PCNA differed in different stages of the cell cycle; it formed distinct subnuclear foci in $S$ phase, while it was distributed in a more diffuse pattern in G2/M phase and post-mitotic phase cells. These subnuclear foci are the sites of active DNA replication, suggesting that replication factories exist in Leishmania, as they do in higher eukaryotes, thus opening avenues for investigating other Leishmania proteins that are involved in DNA replication as part of these replication factories.
DNA replication in eukaryotes is the culmination of events occurring in two distinct stages. In the first stage, origins get licensed to replicate by the establishment of multiprotein complexes called pre-replication complexes (preRCs) at origins in G1 phase, and, in the second stage, these licensed origins get activated, and fire in S phase (Bell \& Dutta, 2002; Diffley, 2004). DNA synthesis of the two strands occurs by two different mechanisms, wherein one strand is synthesized continuously, and the other is synthesized discontinuously. Several enzymes and protein factors are responsible for these processes, and DNA polymerases $\delta$ and $\varepsilon$ play a major role in DNA synthesis. The DNA polymerase processivity factor proliferating cell nuclear antigen (PCNA) considerably augments the processivity of these enzymes (Moldovan et al., 2007; Bravo et al., 1987; Prelich et al., 1987). Replication proteins and their auxiliary factors are organized in complexes

Abbreviations: DAPI, 4',6-diamidino-2-phenylindole; ORC, origin recognition complex; PCNA, proliferating cell nuclear antigen; pre-RC, prereplication complex.

The GenBank/EMBL/DDBJ accession number for the LdPCNA sequence reported in this paper is GQ249893.

A CLUSTALW analysis of LdPCNA and PCNAs from various trypanosomatids is available with the online version of this paper. called replisomes. Several replicons and their replisomes are ordered into clusters called replication factories or foci. Replication foci can be indirectly or directly labelled by short pulses with fluorescently labelled nucleotides, and they are visible by fluorescence microscopy (Lengronne et al., 2001; Pasero et al., 1997; Gilbert, 2001; Kitamura et al., 2006). Each replication focus is believed to harbour several replication forks (Berezney et al., 2000; Cook, 2001).

Replication foci are rich in the enzymes and accessory proteins that are involved in DNA replication. These enzymes and proteins include DNA polymerases, PCNA, replication factor $\mathrm{C}$, replication protein $\mathrm{A}$ and DNA ligases, and cell cycle regulators such as cyclin A (Leonhardt et al., 2000; Cardoso et al., 1993). PCNA was the first protein to be identified at these replication foci, and it has been widely used as a marker for these foci in higher eukaryotes, as it is conserved, and is absolutely essential for DNA replication. Though first identified as the DNA polymerase $\delta$ processivity factor, PCNA forms a trimeric ring around DNA, anchoring not only DNA polymerases, but also interacting with other replication-associated factors, such as Cdt1 and MCM10 (Das-Bradoo et al., 2006; Arias \& Walter, 2006). 
While PCNA has been extensively investigated in higher eukaryotes, among protozoans it has been characterized in Plasmodium falciparum (Patterson et al., 2002; Kilbey et al., 1993; Horrocks et al., 1996; Li et al., 2002) and Toxoplasma gondii (Guerini et al., 2000, 2005) only. In both of these organisms, there are two distinct PCNAs. In T. gondii, one PCNA orthologue remains nuclear throughout the cell cycle, while the second orthologue is nuclear in S phase, but is uniformly distributed throughout the cell in early G1-phase and mitotic cells (Guerini et al., 2005). In P. falciparum, the two PCNAs are expressed during the sexual and asexual stages, though only PfPCNAl has all the conserved motifs of PCNA. PfPCNA1 has been shown to interact with (origin recognition complex) ORC5 and ORC1 of $P$. falciparum, and it co-localizes with both ORC1 and ORC5 in immunofluorescence studies (Gupta et al., 2008, 2009). PfORC1 carries a PIP domain (the domain responsible for docking PCNA-interacting proteins with PCNA), and genetic complementation analysis using a yeast $O R C 1$ mutant strain has revealed that while wild-type PfORC1 can complement mutant ScORC1, PIP mutants of PfORC1 cannot. This finding points to the importance of the PfORC1-PCNA interaction in modulating cell viability, probably through DNA replication.

Not much has been reported about the pre-replication and replication machinery of the genomes of trypanosomatids. The expression of the pre-RC protein ORC1 from Leishmania major has been analysed using GFP-ORC1 (Kumar et al., 2008), and the protein has been found to be nuclear throughout the cell cycle. The pre-RC protein $\mathrm{Cdc} 45$ of Trypanosoma brucei has been demonstrated to interact with BRCA1 of T. brucei (Oyola et al., 2009). Here, we have examined PCNA in the trypanosomatid Leishmania donovani (LdPCNA), and characterized its expression in $L$. donovani promastigotes. LdPCNA was found to be well expressed in proliferating promastigotes. An analysis of the subcellular localization of the protein in relation to the different stages of the cell cycle revealed that, while LdPCNA was nuclear throughout the cell cycle, maximum expression was seen in G1 and S phases. Furthermore, PCNA formed distinct subnuclear foci in S phase. BrdU-labelling experiments indicated that these subnuclear foci were sites of active DNA replication. Our results point towards the existence of replication factories in Leishmania, and indicate that LdPCNA is a promising tool for investigating other Leishmania proteins that are involved in DNA replication as part of these replication factories.

\section{METHODS}

Leishmania culture. L. donovani $1 \mathrm{~S}$ promastigotes were cultured in M199 medium supplemented with $10 \%$ fetal bovine serum, $2 \mathrm{mM}$ L-glutamine, $100 \mu \mathrm{M}$ adenine and $5 \mu \mathrm{g}$ haemin $\mathrm{ml}^{-1}$.

Genomic DNA isolation. About $1 \times 10^{9}$ promastigotes were lysed in $10 \mathrm{mM}$ Tris/ $\mathrm{HCl}$ (pH 8.0), $10 \mathrm{mM}$ EDTA, $150 \mathrm{mM} \mathrm{NaCl}$ and $1 \%$ SDS. After treatment with $100 \mu \mathrm{g}$ proteinase $\mathrm{K} \mathrm{ml}^{-1}$ (Roche), and phenol/chloroform extraction, the genomic DNA was precipitated with ethanol.

Cloning of PCNA. The primers PCNA-F (5'-CACCGGATCCGAATTCATGCTCGAGGCTCAG- $3^{\prime}$ ) and PCNA-R (5'-TACTGCAGTCCCTCCGCATCGTCCACCTTG-3') were used to amplify the PCNA gene from $L$. donovani genomic DNA, using the high-performance proofreading enzyme Phu DNA polymerase (Finnzymes). The PCR product was cloned into the pENTR/D-TOPO vector (Invitrogen), and subcloned into the EcoRI and PstI sites of the expression vector pASK-IBA43plus (IBA BioTAGnology) for overexpression of recombinant LdPCNA.

Overexpression and purification of PCNA. BL21 Codon Plus cells (Stratagene) harbouring plasmid pASK-PCNA were grown to midexponential phase, and expression of PCNA was induced with $200 \mathrm{ng}$ anhydrotetracycline $\mathrm{ml}^{-1}$. Cells were further incubated at $16{ }^{\circ} \mathrm{C}$ for 16-18 h. To assess protein solubility, harvested cells were resuspended in PBS, lysed on ice by sonication, and the lysate was clarified by centrifugation $(10000 \mathrm{~g})$. After solubilization of the pellet in $3 \times$ SDS-sample loading buffer, the lysate and pellet were analysed for PCNA expression by SDS-PAGE. PCNA was purified by affinity chromatography using Strep-Tactin II resin (IBA BioTAGnology), according to the instructions of the manufacturer.

Glutaraldehdye cross-linking. An $8-10 \mu \mathrm{g}$ quantity of purified PCNA was incubated for $5 \mathrm{~min}$ in $10 \mathrm{mM}$ Tris/ $\mathrm{HCl}$ ( $\mathrm{pH} 8.0$ ) containing $0.1 \%$ glutaraldehyde. Reactions were stopped by the addition of $3 \times$ SDS-sample loading buffer, and the product was resolved by SDS-PAGE on $8 \%$ gels.

Raising antibodies to PCNA, and their characterization. Polyclonal antibodies were raised in mouse and rabbit. Antibodies were tested at dilutions of $1: 10000,1: 5000$ and $1: 2500$ by Western blotting, as described by Sambrook et al. (1989).

Preparation of Leishmania extracts. L. donovani whole-cell extracts were prepared using the M-PER kit (Pierce Biotechnology), and nuclear and cytosolic extracts were prepared using the NE-PER kit (Pierce Biotechnology).

Immunofluorescence analysis. For examining the subcellular localization of PCNA, harvested promastigotes were washed with PBS and fixed with $2 \%$ paraformaldehyde for $20 \mathrm{~min}$, and cell spreads were made on poly-lysine-coated coverslips. The cells were permeabilized with $0.1 \%$ Triton X-100 in PBS for $5 \mathrm{~min}$, and then washed, and subjected to blocking with $10 \%$ chicken serum (Invitrogen). After washing, the cells were incubated with antiPCNA antibody (1:100 dilution) for $1-2 \mathrm{~h}$ at room temperature, and then washed, and incubated with Texas-red-labelled anti-mouse secondary antibody (Jackson ImmunoResearch Laboratories). After washing, the coverslips were mounted in anti-fade solution containing 4',6-diamidino-2-phenylindole (DAPI) (Vectashield; Vector Laboratories). Cells were viewed and images were acquired using a $\times 100$ objective, utilizing a motorized epifluorescence microscope (Upright Axioimager M1; Carl Zeiss MicroImaging) equipped with a high-resolution camera (AxioCam MRm Rev. 2; Carl Zeiss MicroImaging). Images were analysed by AxioVision Software Rel. 4.4 (Carl Zeiss MicroImaging).

To quantify PCNA expression, fluorescence intensity of nuclear PCNA was quantified using ImageJ software (Wayne Rasband, National Institutes of Health, Bethesda, MD, USA).

For examining replication foci, exponentially growing promastigotes were pulsed with $40 \mu \mathrm{M}$ BrdU for $20 \mathrm{~min}$. Cells were then harvested, washed with PBS, and fixed in $2 \%$ paraformaldehyde. Cell spreads were permeabilized and blocked, and then incubated with both anti- 
BrdU antibody (GE Healthcare) and rabbit anti-PCNA antibody (1:400 dilution). After washing, Texas-red-labelled anti-mouse (Jackson ImmunoResearch Laboratories) and Alexa-Fluor-488labelled anti-rabbit (Invitrogen) secondary antibodies were applied. After washing, the coverslips were mounted, and imaging of nuclei was done using an inverted motorized confocal microscope (LSM 510; Carl Zeiss MicroImaging) with a $\times 100$ objective by collecting images at high speed in different focal planes ( $Z$ stacks) throughout the entire nucleus.

For examining the distribution pattern of PCNA after exposure to $\mathrm{UV}$, exponentially growing cells were pulsed with BrdU for $10 \mathrm{~min}$, irradiated using a hand-held UV lamp $(254 \mathrm{~nm})$, and further incubated for $10 \mathrm{~min}$. Cells were harvested for immunofluorescence analysis by using BrdU and PCNA antibodies, as described above.

\section{RESULTS AND DISCUSSION}

\section{Cloning and purification of PCNA}

While two PCNA orthologues have been found in each of the other protozoan species that have been studied (Li et al., 2002; Guerini et al., 2005; Patterson et al., 2002), the annotation of the $L$. major whole genome sequence revealed the presence of a single PCNA orthologue (Ivens et al., 2005). Based on the published L. major sequence, we designed end primers to amplify PCNA from L. donovani genomic DNA. The $\sim 0.8 \mathrm{~kb}$ amplicon obtained (Fig. 1a) was cloned, and sequenced from both ends using overlapping primers. Clones of amplicons obtained from two independent PCRs were sequenced. An analysis of the derived amino acid sequence of LdPCNA showed that the protein was 293 aa in length, and had the conserved motifs that typify PCNAs of other eukaryotic organisms (Fig. 1b). A comparative pairwise analysis of the amino acid sequence of LdPCNA with PCNAs from other eukaryotes (Fig. 1c) revealed that LdPCNA shared 55-65\% homology and 30$40 \%$ identity with those PCNAs. When comparing LdPCNA with PCNAs from other trypanosomatids (based on the published genome sequences) (Supplementary Fig. S1), we found that while the protein shared between 95 and $100 \%$ identity with PCNAs from other Leishmania species, it shared about $75 \%$ identity with PCNAs of T. brucei and Trypanosoma cruzi. The C-terminal insertion apparent in Fig. 1(c) is conserved in all the trypanosomatids.

In order to examine PCNA expression in L. donovani promastigotes, LdPCNA was overexpressed in Escherichia coli, the recombinant protein was purified, and antibodies were raised against it. The gene was subcloned into the $E$. coli expression vector pASK-IBA43plus, and the protein was overexpressed in E. coli BL21 Codon Plus cells (Fig. 2a). The overexpressed protein was about $60 \%$ soluble (Fig. 2b), and it was purified by affinity chromatography using a Strep-Tactin II matrix. The pure protein (Fig. 2c) was found to exist as a trimer by using glutaraldehyde cross-linking (Fig. 2d). This result is in keeping with the presence of the conserved residues responsible for trimer intersubunit interactions in PCNA (Fig. 1b). All eukaryotic PCNAs reported to date form a ring-shaped homotrimeric complex that encircles DNA, and form a sliding clamp (Krishna et al., 1994; Gulbis et al., 1996; Bowman et al., 2004).

\section{PCNA is well-expressed in actively proliferating Leishmania promastigotes}

Polyclonal antibodies against LdPCNA were raised in mice, and subsequently in rabbits. To assess the specificity and efficacy of the antibodies raised, Western blotting analysis was carried out using recombinant LdPCNA. While preimmune serum did not react with recombinant LdPCNA, anti-PCNA antiserum detected as little as $1 \mathrm{ng}$ LdPCNA at a dilution of 1:5000 (Fig. 3a). The expression of PCNA in $L$. donovani promastigotes was analysed in whole-cell extracts of actively proliferating promastigotes using these antibodies. A single band of the expected size was detected (recombinant LdPCNA is $2 \mathrm{kDa}$ larger because of the tags at both ends), indicating that PCNA was well expressed and that the antibodies were of high specificity (Fig. 3b). In nuclear and cytosolic extracts made from asynchronous cultures of these promastigotes, PCNA was detected in the nuclear extracts only (Fig. 3c), indicating that the protein is primarily nuclear in nature.

\section{PCNA is nuclear throughout the cell cycle, but is mainly expressed in $\mathbf{G} 1$ and $\mathbf{S}$ phases}

The subcellular localization of LdPCNA was examined to see if the specific import of PCNA into the nucleus in only G1 and S phases could be a means of regulation of DNA replication in Leishmania promastigotes. Immunofluorescence analysis of cells harvested from asynchronous procyclic cultures was carried out using the anti-PCNA antibodies we raised. In trypanosomatids, due to the staggered timing of replication of kinetoplast DNA as compared with the genome, the kinetoplast can be used as a marker for cell cycle stage of an individual cell (Siegel $e t$ al., 2008). While cells in G1 phase contain one nucleus and one short roundish kinetoplast, S-phase cells contain one nucleus and one elongated kinetoplast. Cells in G2/M phase contain one nucleus and two kinetoplasts, as kinetoplast replication is complete by this stage of the cell cycle, and the newly synthesized kinetoplasts are already separate. Post-mitotic cells have two completely separated nuclei and two kinetoplasts. Indirect immunofluorescence studies with anti-PCNA antibodies revealed that this protein was nuclear at all stages of the cell cycle (Fig. 4a). We were unable to detect PCNA in the cytoplasm in any phase of cell cycle. However, in over 400 cells examined, expression was always robust in G1- and S-phase cells, but very weak in mitotic cells (Fig. 4a). Quantification of fluorescence intensity of nuclear PCNA at different stages of the cell cycle revealed that expression in G2/M phase was about 2.5- to 3-fold lower than in G1 or S phase (Fig. 4b). Post-mitotic cells showed almost as much expression of PCNA as G1- and S-phase cells, suggesting that while PCNA probably degrades during G2/M phase, levels of the 

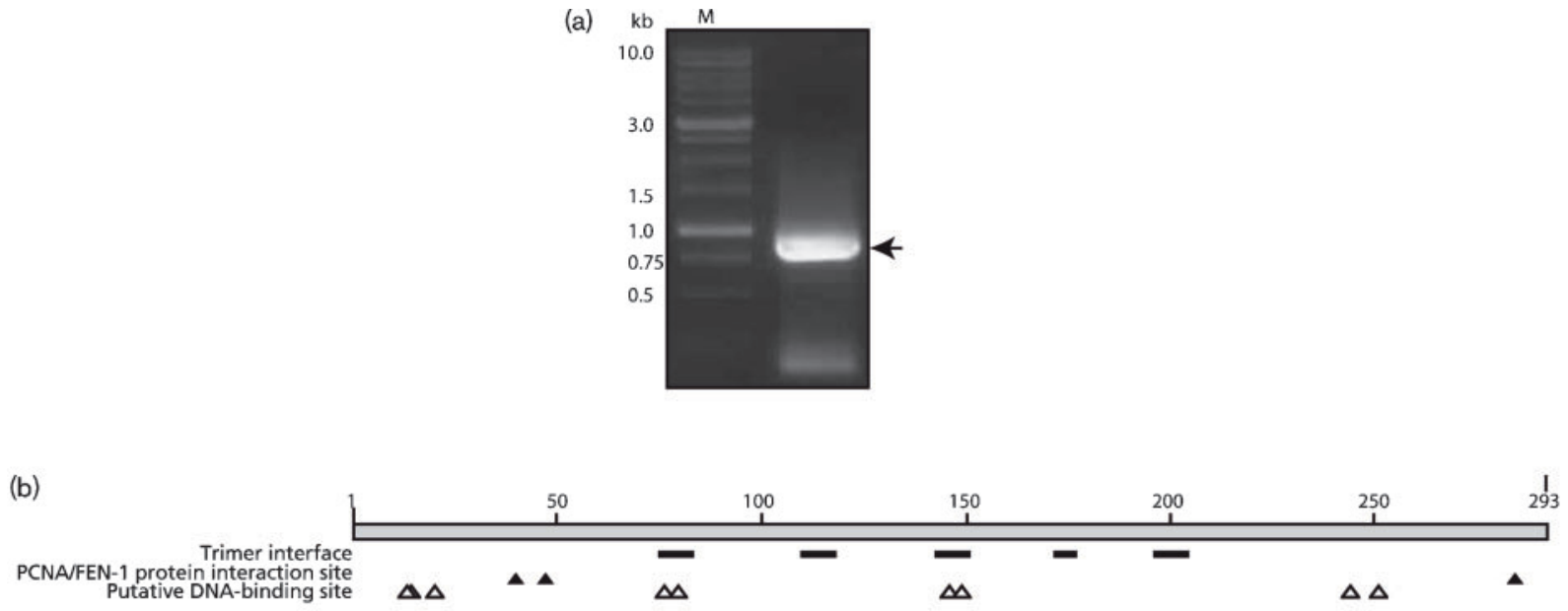

(c)

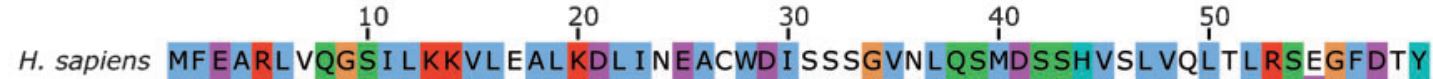

$X$. laevis MFEARLVQGSI L KKVLEAL KDL I DEACWD I TSSG I SLQSMDSSHVSL VQLTLRSDGFDTY

D. melanogaster MFEARLGQAT I L KKI LDA I KDL LNEATFDCSDSG I QLQAMDNSHVSLVSLTLRSDGFDKF

S. pombe MLEARFQQAALL KKLLDAI KEL VTDANFDCNDNG I SLQAMDSSHVAL VSML I KSDGFEPY

S. cerevisiae MLEAKFEEASLFKRI I DGF KDCVQLVNFQCKEDG I I AQAVDDSRVLLVSLEI GVEAFQEY

L. donovani MLEAQVQYASLWKRLVEC I NGL VNEANFDCNPGGLSI QAMDSSHVALVHML LRDDCFVKY

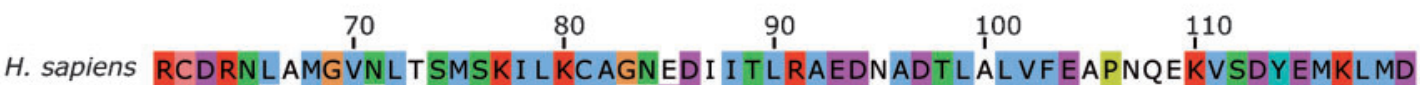

$x$. laevis RCDRNQSI GVKMSSMSKI L KCAASDD I I TLRAEDNADT VTMVF ESPNQEKVSDYEMKLMD

D. melanogaster RCDRNLSMGMNLGSMAKI L KCANNEDNVTMKAQDNADTVT I MF ESANQEKVSDYEMKLMN

S. pombe RCDRNI ALGINLNALSKVLRCAQNEDL VTLKAEDTPEVLNLVFESEKNDRI SDYDVKLMD

S. cerevisiae RCDHPVTLGMDLTSLSKI LRCGNNTDTLTLI ADNTPDS I I LLFEDTKKDR I AEYSL KLMD

L. donovani QCERN I I LGLNLASLSKVLKI VDGNDSL I LRHDDDSDVVTLT SENPEKTRKCEYQL KLLE

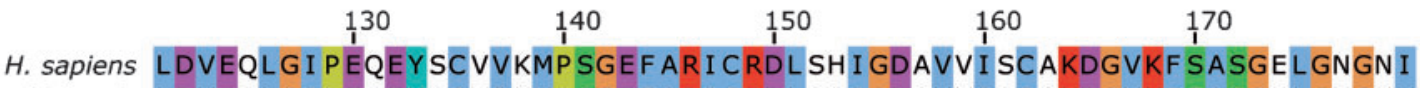

x. laevis LDVEQLGI PEQEYSCVI KMPSGEFAR I CRDLSQI GDAVVI SCAKDGVKF SASGELGTGNV

D. melanogaster LDQEHLG I PETDFSCVVRMPAMEFAR I CRDLAQFSESVVI CCTKEGVKF SASGDVGTAN I

S. pombe I DQEHLGI PD I EYDAT I TMPAAEFQR I TRDLLTLSDSVT INASKEGVRFSCKGDIGNGST

S. cerevisiae I DADF LKI EELQYDSTLSLPSSEFSKI VRDLSQLSDS IN I MI TKET I KFVADGDI GSGSV

L. donovani I EAESMG I PEMDYRSTVTLNSAEFAKI VRDMQVFGDTVT I A I SKEGVKF SSSGDVGQGYT

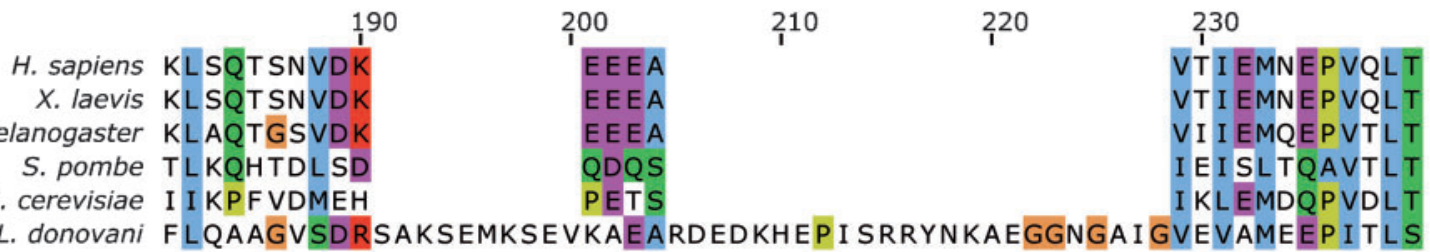

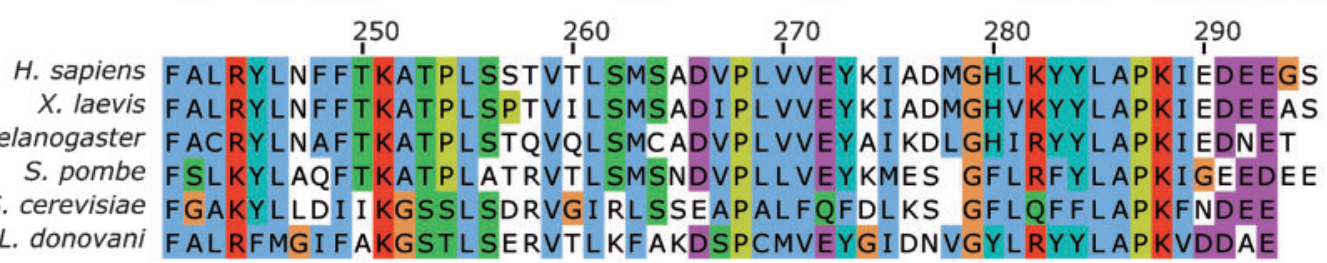

Fig. 1. Cloning of PCNA from L. donovani. (a) Amplification of PCNA from $L$. donovani genomic DNA. The $\sim 0.8 \mathrm{~kb} P C N A$ gene product is indicated by an arrow. M, DNA marker. (b) Schematic representation of the conserved domains in LdPCNA. (c) CLUSTALW (www.ebi.ac.uk/Tools/clustalw2) analysis of LdPCNA and PCNAs from other eukaryotes viewed using the Jalview multiple alignment editor (Waterhouse et al., 2009). H. sapiens, Homo sapiens; X. laevis, Xenopus laevis; D. melanogaster, Drosophila melanogaster; S. pombe, Schizosaccharomyces pombe; S. cerevisiae, Saccharomyces cerevisiae. 
(a)

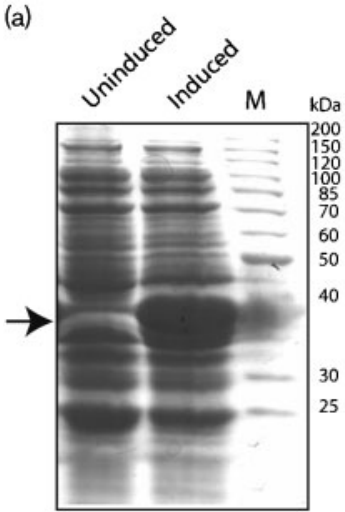

(b)

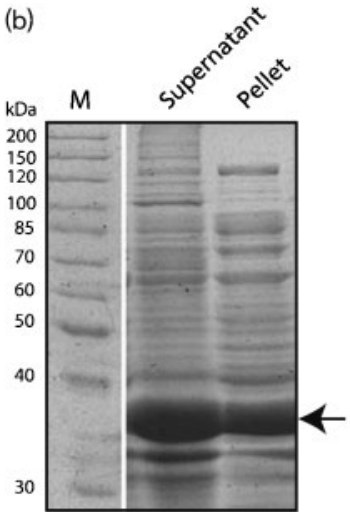

(c)

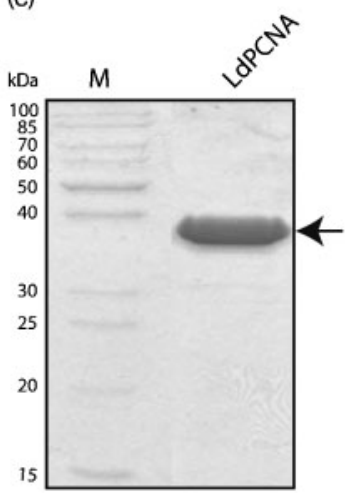

(d)

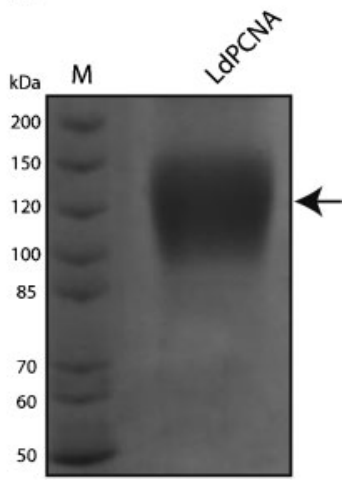

Fig. 2. L. donovani PCNA exists as a trimer. (a) Overexpression of recombinant LdPCNA in E. coli. The arrow indicates overexpressed PCNA. (b) Analysis of solubility of overexpressed LdPCNA (His-PCNA-Strep). (c) SDS-PAGE (12\% gel) analysis of purified LdPCNA (indicated by an arrow) obtained after Strep-Tactin II chromatography. (d) SDS-PAGE (8\% gel) analysis of glutaraldehyde cross-linked purified LdPCNA. The band corresponding to trimeric LdPCNA is indicated by an arrow.

protein ramp up rapidly after nuclear division is complete. PCNA continued to be robustly expressed in metacyclic forms (stage determined by the shape of the parasites, flagellum length and formation of rosettes) (bottom panel, Fig. 4a).

\section{PCNA forms distinct foci within the nucleus of S phase cells, and these foci are the sites of active DNA replication}

The distribution pattern of LdPCNA within the nuclei at different stages of the cell cycle was examined in immunofluorescence analyses by collecting images at high speed in different focal planes ( $Z$ stacks) throughout the entire nucleus. The equatorial sections through the nuclei are shown in Fig. 5. We found that while the distribution of PCNA was more diffuse in pattern in G2/M-phase and post-mitotic cells, distinct foci were evident in S-phase nuclei, suggestive of the fact that replication factories or foci may exist in Leishmania promastigotes, as have been shown in yeasts and higher eukaryotes. G1 cells also showed foci, though not as distinct as those in S-phase cells. To verify that the foci observed in S-phase nuclei were indeed sites of active DNA replication, promastigotes were briefly pulsed with BrdU, and then examined by indirect immunofluorescence for PCNA and BrdU localization patterns within S-phase nuclei. As is evident from Fig. 6(a), BrdU foci colocalized with PCNA foci, implying that replication factories exist even in a protozoan such as Leishmania. PCNA may thus serve as a marker for sites of active DNA replication in this parasite.

Upon exposing the cells to UV radiation, we observed a change in PCNA distribution patterns. Cells were pulsed with BrdU for $10 \mathrm{~min}$, irradiated with a UV lamp $(254 \mathrm{~nm})$, and further incubated in the continued presence of BrdU, for $10 \mathrm{~min}$. Examination of these cells revealed that while some PCNA foci colocalized with BrdU foci, some distinct PCNA foci that did not colocalize with BrdU (a)

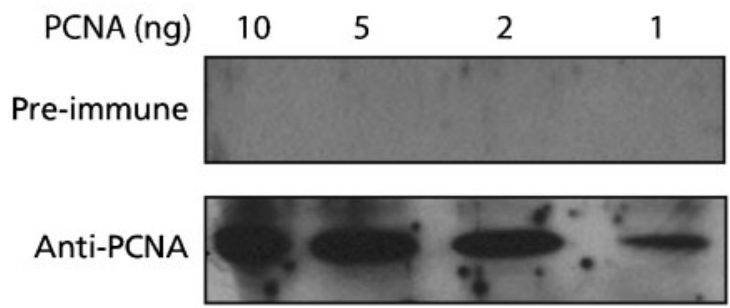

(b)

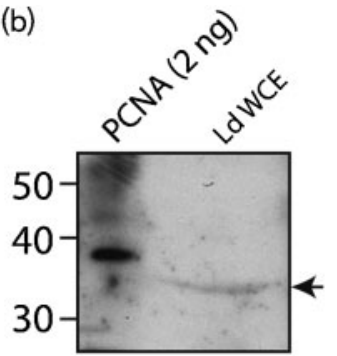

(c)

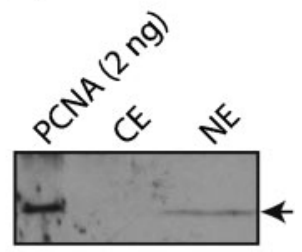

Fig. 3. LdPCNA is expressed in actively proliferating promastigotes, and is mainly nuclear in nature. (a) Western blot analysis of recombinant LdPCNA (amounts as indicated) with mouse anti-PCNA antibody used at a dilution of 1:5000. (b) Whole-cell extract (WCE; $60 \mu \mathrm{g}$ ) obtained from L. donovani promastigotes resolved on SDS-PAGE (12\% gel), followed by Western blotting using anti-PCNA antibodies at a dilution of $1: 2000$. (c) Analysis of $L$. donovani nuclear extract (NE) and cytosolic extract (CE) by SDS-PAGE (10\% gel), followed by Western blotting using the anti-PCNA antibodies at a dilution of $1: 2000$. 
(a)
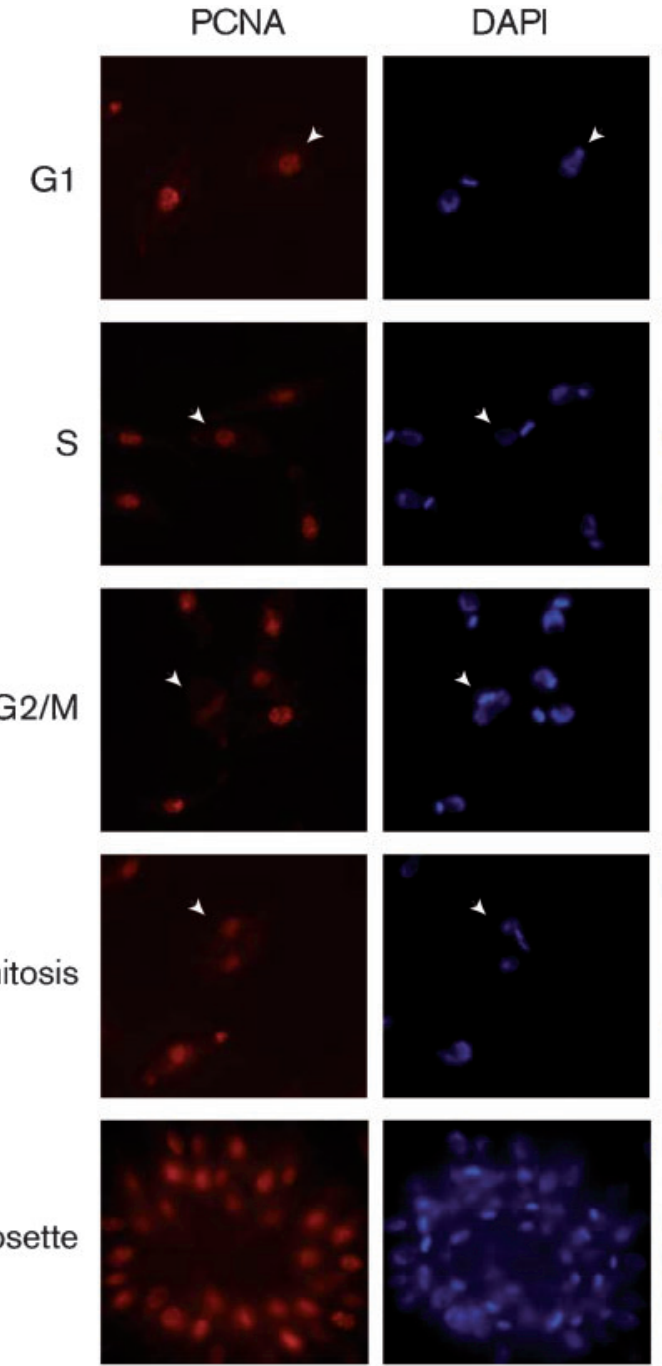

(b)

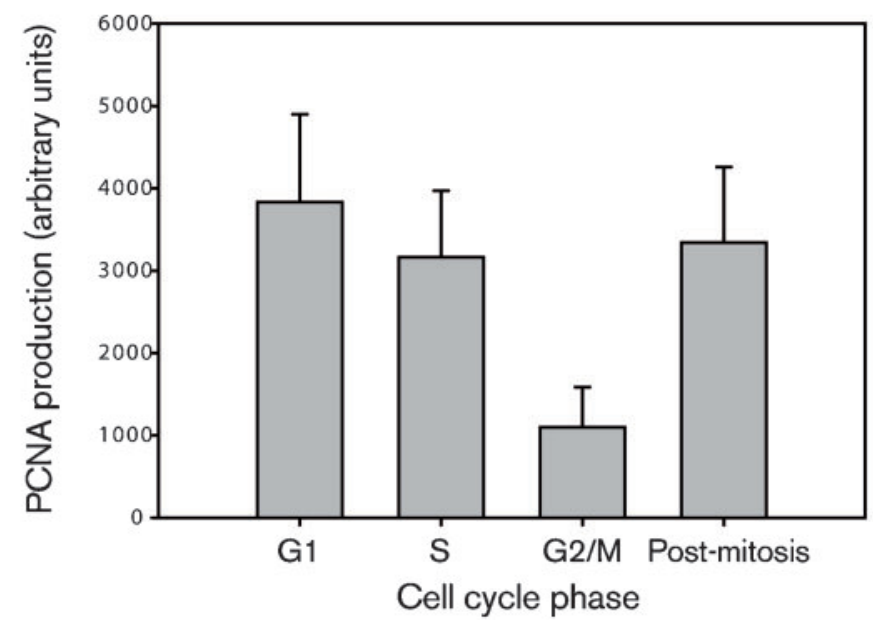

Fig. 4. PCNA is nuclear throughout the cell cycle, and is strongly expressed in G1- and Sphase cells. (a) G1-phase cells: one nucleus, one short kinetoplast. S-phase cells: one nucleus, one elongated kinetoplast. G2/Mphase cells: one nucleus, two kinetoplasts. Post-mitosis cells: two nuclei, two kinetoplasts. Rosette formation is a hallmark of the metacyclic stage. Arrowheads indicate the cells representing the specific stage of the cell cycle. Cells were visualized using an epifluorescence microscope, as described. (b) Quantification of nuclear expression of PCNA by ImageJ software. The images of 10 cells of each cell type were analysed. Error bars indicate SD. foci were also observed (Fig. 6b; upper panel). In some cells, the pattern of PCNA was more diffuse upon UV irradiation (Fig. 6b; lower panel), and PCNA largely did not colocalize with BrdU in these cells. These results are in keeping with earlier findings in mammalian cells that demonstrated that upon induction of DNA damage by 

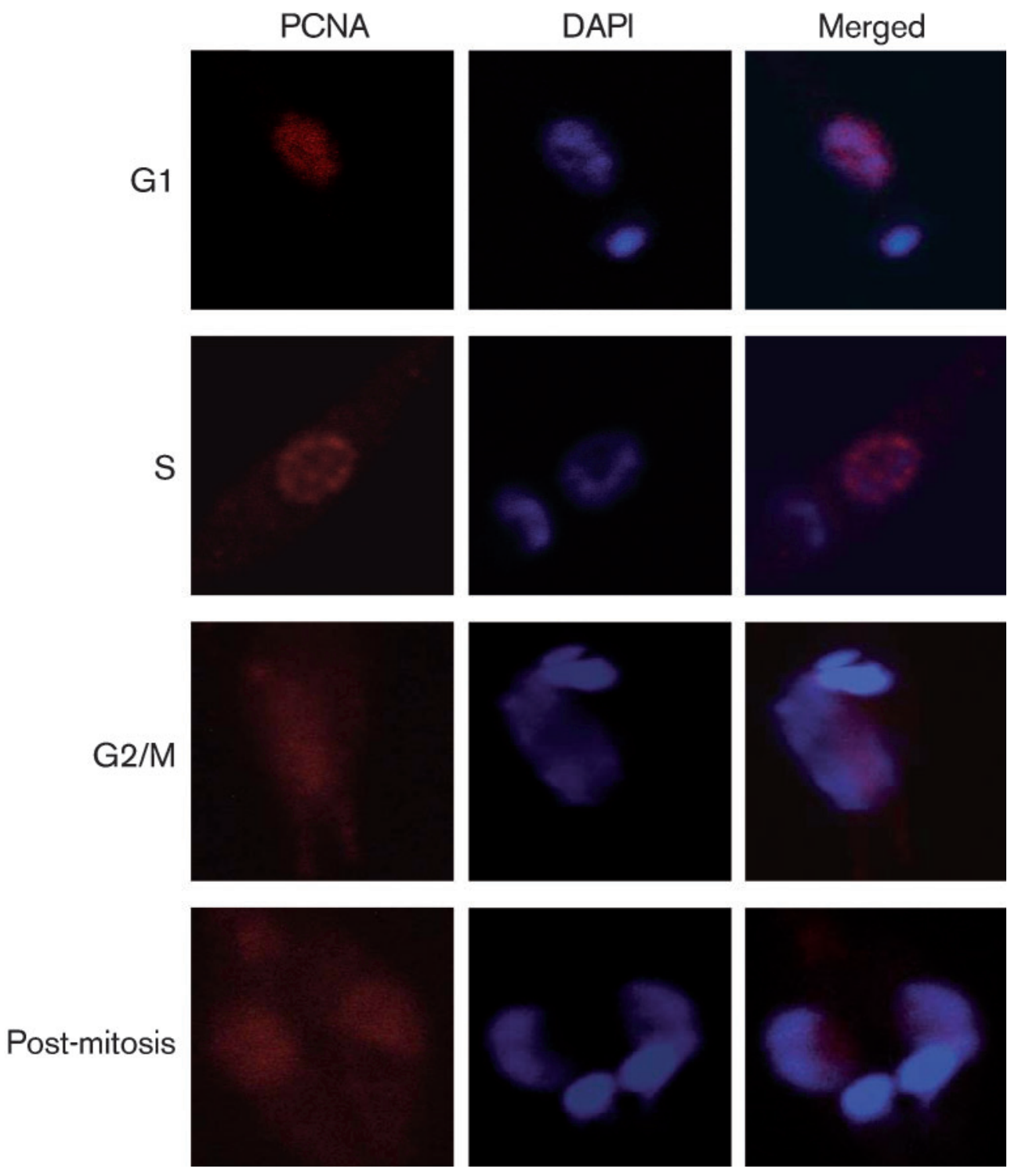

Fig. 5. PCNA distribution patterns vary with the progress of cell cycle. Cells were immunostained with mouse anti-PCNA antibody (1:100 dilution) and DAPI. The nuclei of cells in different stages of the cell cycle were analysed by collecting $Z$ stack images using a confocal microscope, as described.

exposure to UV radiation, PCNA is recruited to the sites of damage (Solomon et al., 2004; Essers et al., 2005).

Replication foci have been shown in yeasts and higher eukaryotes. The dynamics of these foci have been studied in Schizosaccharomyces pombe and mammalian cells (Leonhardt et al., 2000; Somanathan et al., 2001; Sporbert et al., 2002; Solomon et al., 2004; Essers et al., 2005; Meister et al., 2007). The pattern of these foci changes with S-phase progression. While a greater number of foci are apparent in early and mid S phase, fewer foci are seen in late S phase (Meister et al., 2007). These changes are believed to mirror the pattern of DNA synthesis in S phase, with transcriptionally active euchromatin replicated early in S phase, and heterochromatic DNA replicated later on.
Eukaryotic DNA replication occurs by the activation of multiple origins that are selected by the assembly of multiprotein pre-RCs. Pre-RCs assemble by the initial association of ORCs 1-6 to origin DNA, followed by the sequential association of Cdc6, Cdt1, Mcms2-7 and Cdc45 (Bell \& Dutta, 2002). As cells enter S phase, cyclindependent kinases and Cdc7-Dbf4 kinase target the pre-RC proteins, and essential replication factors, such as Cdc45, initiate a series of events culminating in DNA synthesis (Zou \& Stillman, 1998, 2000; Sclafani, 2000; Bell \& Dutta, 2002; Sheu \& Stillman, 2006). Though the replication machinery of Leishmania resembles that of higher eukaryotes, significant differences have been implicated from the analysis of its genome (Ivens et al., 2005); this revealed the 
(a)
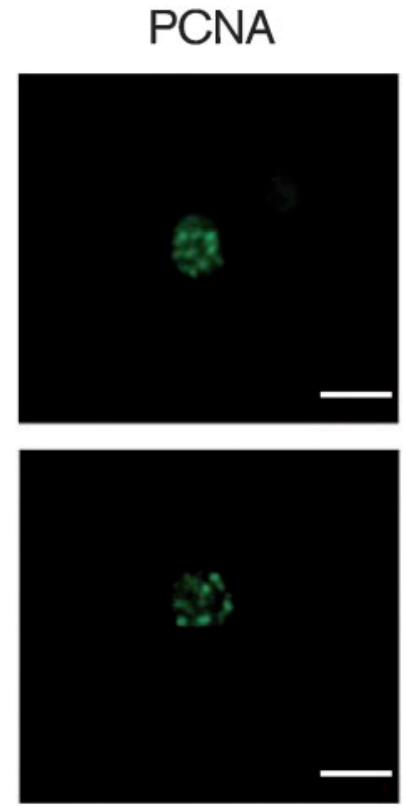

(b)
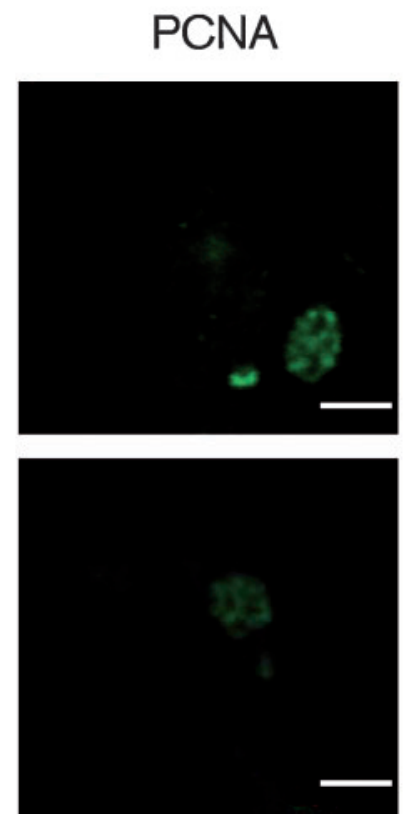
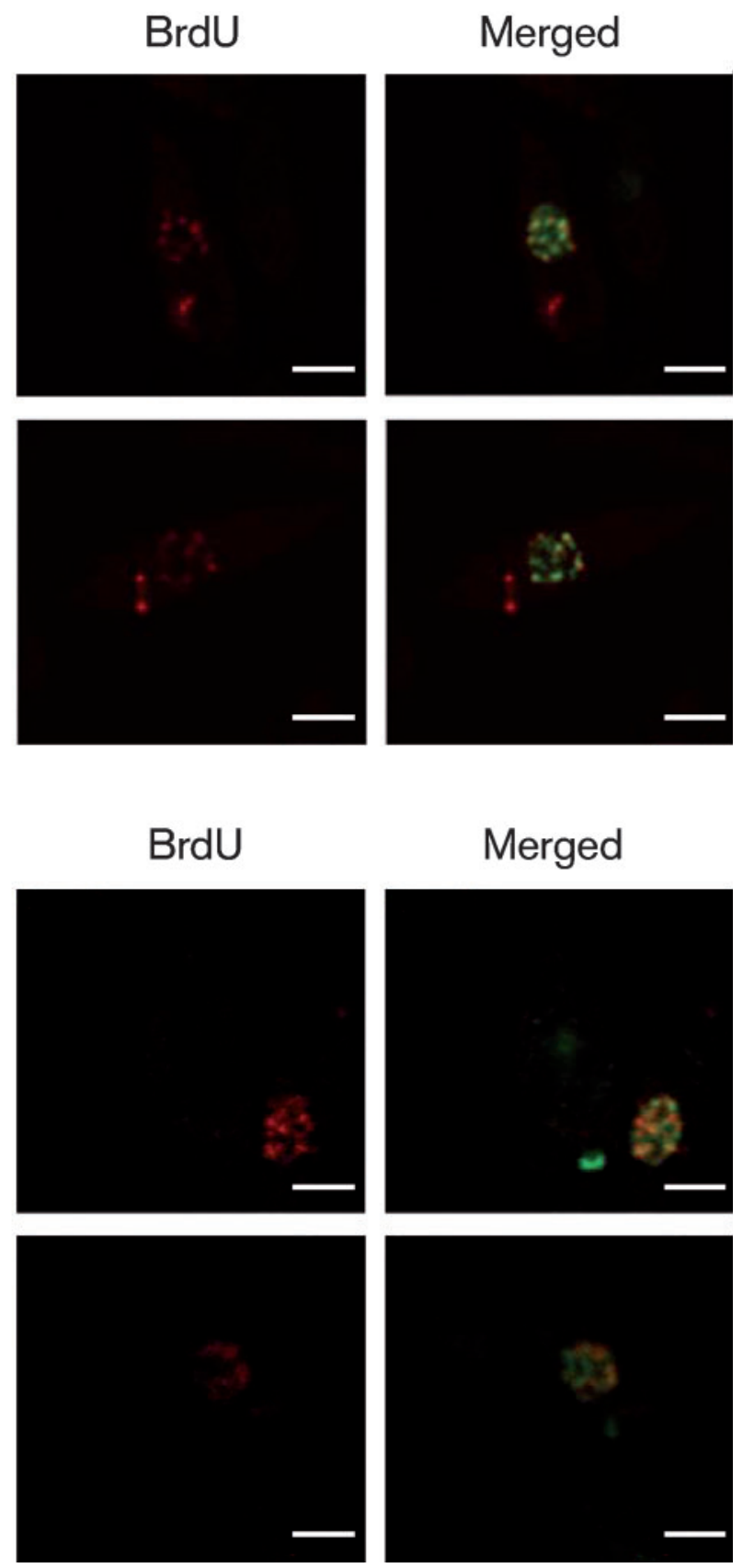

Fig. 6. (a) Actively replicating DNA sites colocalize with PCNA foci. S-phase cells were doubly immunostained with rabbit antiPCNA antibody (1:400 dilution) and mouse monoclonal anti-BrdU antibody (according to the instructions of the manufacturer). Actively proliferating Leishmania promastigotes were pulsed with BrdU for 20 min prior to harvesting and fixing cells for immunofluorescence. S-phase cells were labelled with BrdU. The nuclei of BrdU labelled cells were analysed by collecting Z stack images using a confocal microscope, as described. Bars, $2 \mu \mathrm{m}$. (b) S-phase cells, sampled 10 min after UV irradiation and doubly stained with rabbit anti-PCNA antibody ( $1: 400$ dilution) and mouse monoclonal anti-BrdU antibody (according to the instructions of the manufacturer).

presence of only one ORC orthologue (ORC1), and orthologues of MCMs2-7 and Cdc45. Orthologues of Cdt1, MCM10, Dbf4 or Cdc7 have not been annotated thus far. The absence of several eukaryotic orthologues of pre-
RC proteins in Leishmania suggests that, in this organism, novel unidentified proteins may be involved in replication initiation. These proteins are likely to be a part of the replication factories in $\mathrm{S}$ phase. LdPCNA can serve as a 
marker for these factories, and can be utilized to seek out other Leishmania replication proteins, including any novel proteins that may be involved in the process.

\section{ACKNOWLEDGEMENTS}

We thank Dr Vinay Nandicoori and Dr Sagar Sengupta for the use of the fluorescence microscope, and the laboratory of Dr Deepak Pental for DNA sequencing. This work was supported by a grant from the Council of Scientific and Industrial Research, India, and the Department of Biotechnology, India. D. K. is a Senior Research Fellow supported from this grant. N.M. is a University Grants Commission Senior Research Fellow.

\section{REFERENCES}

Arias, E. E. \& Walter, J. C. (2006). PCNA functions as a molecular platform to trigger Cdt1 destruction and prevent re-replication. Nat Cell Biol 8, 84-90.

Bell, S. P. \& Dutta, A. (2002). DNA replication in eukaryotic cells. Annu Rev Biochem 71, 333-374.

Berezney, R., Dubey, D. D. \& Huberman, J. A. (2000). Heterogeneity of eukaryotic replicons, replicon clusters, and replication foci. Chromosoma 108, 471-484.

Bowman, G. D., O'Donnell, M. \& Kuriyan, J. (2004). Structural analysis of a eukaryotic sliding DNA clamp-clamp loader complex. Nature 429, 724-730.

Bravo, R., Frank, R., Blundell, P. A. \& Macdonald-Bravo, H. (1987), Cyclin/PCNA is the auxiliary protein of DNA polymerase- $\delta$. Nature 326, 515-517.

Cardoso, M. C., Leonhardt, H. \& Nadal-Ginard, B. (1993). Reversal of terminal differentiation and control of DNA replication: cyclin A and Cdk2 specifically localize at subnuclear sites of DNA replication. Cell 74, 979-992.

Cook, P. R. (2001). Principles of Nuclear Structures and Function. New York: Wiley-Liss.

Das-Bradoo, S., Ricke, R. M. \& Bielinsky, A. K. (2006). Interaction between PCNA and diubiquitinated $\mathrm{Mcm} 10$ is essential for cell growth in budding yeast. Mol Cell Biol 26, 4806-4817.

Diffley, J. F. (2004). Regulation of early events in chromosome replication. Curr Biol 14, R778-R786.

Essers, J., Theil, A. F., Baldeyron, C., van Cappellen, W. A. Houtsmuller, A. B., Kanaar, R. \& Vermeulen, W. (2005). Nuclear dynamics of PCNA in DNA replication and repair. Mol Cell Biol 25, 9350-9359.

Gilbert, D. M. (2001). Making sense of eukaryotic DNA replication origins. Science 294, 96-100.

Guerini, M. N., Que, X., Reed, S. L. \& White, M. W. (2000). Two genes encoding unique proliferating-cell-nuclear-antigens are expressed in Toxoplasma gondii. Mol Biochem Parasitol 109, 121-131.

Guerini, M. N., Behnke, M. S. \& White, M. W. (2005). Biochemical and genetic analysis of the distinct proliferating cell nuclear antigens of Toxoplasma gondii. Mol Biochem Parasitol 142, 56-65.

Gulbis, J. M., Kelman, Z., Hurwitz, J., O’Donnell, M. \& Kuriyan, J. (1996). Structure of the C-terminal region of p21(WAF1/CIP1) complexed with human PCNA. Cell 87, 297-306.

Gupta, A., Mehra, P. \& Dhar, S. K. (2008). Plasmodium falciparum origin recognition complex subunit 5: functional characterization and role in DNA replication foci formation. Mol Microbiol 69, 646-665.
Gupta, A., Mehra, P., Deshmukh, A., Dar, A., Mitra, P., Roy, N. \& Dhar, S. K. (2009). Functional dissection of the catalytic carboxyl-terminal domain of human malaria parasite Plasmodium falciparum origin recognition complex subunit 1 (PfORC1). Eukaryot Cell 8, 13411351.

Horrocks, P., Jackson, M., Cheesman, S., White, J. H. \& Kilbey, B. J. (1996). Stage specific expression of proliferating cell nuclear antigen and DNA polymerase $\delta$ from Plasmodium falciparum. Mol Biochem Parasitol 79, 177-182.

Ivens, A. C., Peacock, C. S., Worthey, E. A., Murphy, L., Aggarwal, G., Berriman, M., Sisk, E., Rajandream, M. A., Adlem, E. \& other authors (2005). The genome of the kinetoplastid parasite, Leishmania major. Science 309, 436-442.

Kilbey, B. J., Fraser, I., McAleese, S., Goman, M. \& Ridley, R. G. (1993). Molecular characterisation and stage-specific expression of proliferating cell nuclear antigen (PCNA) from the malarial parasite, Plasmodium falciparum. Nucleic Acids Res 21, 239-243.

Kitamura, E., Blow, J. J. \& Tanaka, T. U. (2006). Live-cell imaging reveals replication of individual replicons in eukaryotic replication factories. Cell 125, 1297-1308.

Krishna, T. S., Kong, X. P., Gary, S., Burgers, P. M. \& Kuriyan, J. (1994). Crystal structure of the eukaryotic DNA polymerase processivity factor PCNA. Cell 79, 1233-1243.

Kumar, D., Mukherji, A. \& Saha, S. (2008). Expression and subcellular localization of ORC1 in Leishmania major. Biochem Biophys Res Commun 375, 74-79.

Lengronne, A., Pasero, P., Bensimon, A. \& Schwob, E. (2001). Monitoring $S$ phase progression globally and locally using BrdU incorporation in TK $(+)$ yeast strains. Nucleic Acids Res 29, 14331442.

Leonhardt, H., Rahn, H. P., Weinzierl, P., Sporbert, A., Cremer, T., Zink, D. \& Cardoso, M. C. (2000). Dynamics of DNA replication factories in living cells. J Cell Biol 149, 271-280.

Li, J. L., Warren, A. V. \& Cox, L. S. (2002). Identification of a second proliferating cell nuclear antigen in the human malarial pathogen Plasmodium falciparum. Int J Parasitol 32, 1683-1692.

Meister, P., Taddei, A., Ponti, A., Baldacci, G. \& Gasser, S. M. (2007). Replication foci dynamics: replication patterns are modulated by S-phase checkpoint kinases in fission yeast. EMBO J 26, 13151326.

Moldovan, G. L., Pfander, B. \& Jentsch, S. (2007). PCNA, the maestro of the replication fork. Cell 129, 665-679.

Oyola, S. O., Bringaud, F. \& Melville, S. E. (2009). A kinetoplastid BRCA2 interacts with DNA replication protein CDC45. Int J Parasitol 39, 59-69.

Pasero, P., Braguglia, D. \& Gasser, S. M. (1997). ORC-dependent and origin-specific initiation of DNA replication at defined foci in isolated yeast nuclei. Genes Dev 11, 1504-1518.

Patterson, S., Whittle, C., Robert, C. \& Chakrabarti, D. (2002). Molecular characterization and expression of an alternate proliferating cell nuclear antigen homologue, PfPCNA2, in Plasmodium falciparum. Biochem Biophys Res Commun 298, 371-376.

Prelich, G., Tan, C. K., Kostura, M., Mathews, M. B., So, A. G., Downey, K. M. \& Stillman, B. (1987). Functional identity of proliferating cell nuclear antigen and a DNA polymerase- $\delta$ auxiliary protein. Nature 326, 517-520.

Sambrook, J., Fristch, E. F. \& Maniatis, T. (1989). Molecular Cloning: a Laboratory Manual, 2nd edn. Cold Spring Harbor, NY: Cold Spring Harbor Laboratory.

Sclafani, R. A. (2000). Cdc7p-Dbf4p becomes famous in the cell cycle. J Cell Sci 113, 2111-2117. 
Sheu, Y. J. \& Stillman, B. (2006). Cdc7-Dbf4 phosphorylates MCM proteins via a docking site-mediated mechanism to promote $S$ phase progression. Mol Cell 24, 101-113.

Siegel, T. N., Hekstra, D. R. \& Cross, G. A. (2008). Analysis of the Trypanosoma brucei cell cycle by quantitative DAPI imaging. Mol Biochem Parasitol 160, 171-174.

Solomon, D. A., Cardoso, M. C. \& Knudsen, E. S. (2004). Dynamic targeting of the replication machinery to sites of DNA damage. J Cell Biol 166, 455-463.

Somanathan, S., Suchyna, T. M., Siegel, A. J. \& Berezney, R. (2001). Targeting of PCNA to sites of DNA replication in the mammalian cell nucleus. J Cell Biochem 81, 56-67.

Sporbert, A., Gahl, A., Ankerhold, R., Leonhardt, H. \& Cardoso, M. C. (2002). DNA polymerase clamp shows little turnover at established replication sites but sequential de novo assembly at adjacent origin clusters. Mol Cell 10, 1355-1365.

Waterhouse, A. M., Procter, J. B., Martin, D. M. A., Clamp, M. \& Barton, G. J. (2009). Jalview Version 2 - a multiple sequence alignment editor and analysis workbench. Bioinformatics 25, 1189-1191.

Zou, L. \& Stillman, B. (1998). Formation of a preinitiation complex by $\mathrm{S}$-phase cyclin CDK-dependent loading of Cdc45p onto chromatin. Science 280, 593-596.

Zou, L. \& Stillman, B. (2000). Assembly of a complex containing $\mathrm{Cdc45p}$, replication protein $\mathrm{A}$, and $\mathrm{Mcm} 2 \mathrm{p}$ at replication origins controlled by S-phase cyclin-dependent kinases and Cdc7p-Dbf4p kinase. Mol Cell Biol 20, 3086-3096.

Edited by: L. Knoll 